\title{
Identification of Nonlinear Behavior of an Electromechanical Loudspeaker; Experimental Approach
}

\author{
Naser Elmi and Navid Azadi \\ Amirkabir University of Technology, Mechanical Engineering Department, 15875-4413, Tehran, Iran.
}

(Received 22 February 2015; accepted 10 June 2016)

The performance of common linear algorithms in active noise control applications can be degenerated mainly due to the unmodeled nonlinearities of loudspeakers as actuators in noise attenuation process. The aim of this article is to propose different methods such as prediction error method (PEM), nonlinear autoregressive network with exogenous input (NARX), and series-parallel NARX network based on neural network to experimentally identify the nonlinear behaviour of a loudspeaker. A model of loudspeaker is being used in noise cancellation and control algorithms; hence, its validity and robustness to input amplitude and frequency plays a crucial role in noise control. The results of this article, which are completely based on real and experimental data, demonstrate that neural network based series-parallel NARX model is the best estimator for the fully nonlinear behaviour of the loudspeaker. The capability and robustness of this estimator in comparison with other methods is examined by different test inputs with different amplitudes and frequencies.

\section{INTRODUCTION}

The first dynamic model to describe the electro dynamic loudspeaker was proposed by Thiele and Small. ${ }^{1}$ Their model utilized linear equations to present loudspeaker dynamics. However, a loudspeaker shows nonlinearities that produce distortions. In previous studies, ${ }^{2,3}$ the nonlinearities of the loudspeaker dynamic are categorized in three main sources: suspensions, diaphragms, and motors. Furthermore, many attempts have been done to model and study the nonlinearities of the loudspeaker dynamic. For instance, in the most recent studies, Ravaund et al. proposed a time varying third order model to describe the dynamic of an electro dynamic loudspeaker. ${ }^{4}$ Also, in other study, they ranked the nonlinearities of loudspeaker based on their importance for different input magnitudes and frequencies. ${ }^{5}$ On the other hand, Faifer et al. suggested a novel method to estimate nonlinear loudspeaker parameters such as stiffness by linearizing the nonlinear dynamic of the loudspeaker. ${ }^{6}$ Chang et al. used experimental laser vibrometry and Finite Element Analysis to model the nonlinear stiffness of the diaphragm. ${ }^{7}$ Also, to measure the total harmonic distortion, the electroacoustic system is modelled by its equivalent electrical circuit. In another research, the significant importance of coil position in improving sound quality and reduction of total harmonic distortion is studied using a nonlinear mathematical model of a loudspeaker. ${ }^{8}$ Considering all the above-mentioned studies, we observe that all of them have used grey- box models, and therefore they are completely limited to the amplitude and the frequency of input signal. In other words, big input signal such as big electrical current can excite the nonlinear oscillatory behaviours of the diaphragm of loudspeakers; therefore, simple grey- box models would not be accurate enough to demonstrate its behaviour.

Although passive noise and vibration control have been successfully implemented in many engineering applications such as printed circuit boards, ${ }^{9}, 10$ jet-impingement cooling systems, ${ }^{11-14}$ optic devices, ${ }^{15}$ and cryogenic coolers, ${ }^{16}$ active noise control (ANC) is necessary in many other applications where some conditions like randomness or non- stationary behaviour of excitation renders passive techniques ineffective. Loudspeakers play a crucial role in active noise control. However, Azadi and Ohadi have claimed that neglecting the nonlinearities of loudspeaker can deteriorate ANC performance. ${ }^{17}$ Therefore, Azadi et al. used neural networks for modelling the loudspeaker in the filtered gradient active fuzzy neural network noise control in an enclosure. ${ }^{18}$ The aforementioned researches demonstrate the vital need to use black-box models to model the nonlinear behaviour of loudspeaker especially in ANC application. ${ }^{18,19}$ Therefore, in this article, the authors will use different system identification techniques to model loudspeaker dynamics in an offline manner with experimental data. The most essential advantage of using offline identification is the reduction of calculation load and increasing the speed of ANC system. Note that, a comprehensive and accurate identified model of loudspeaker in offline condition can be used in ANC application by considering possible model variations as model disturbance and uncertainty. In order to do so, different methods will be used and tested with a variety of input voltage amplitudes and frequencies.

\section{LINEAR AND NONLINEAR MODEL OF LOUDSPEAKER}

As stated earlier in Sec. 1, a loudspeaker may exhibit nonlinear behaviour in certain working condition, such as low frequency and/or high amplitude input signals. One of the characteristics of a linear system is that when a pure sine signal is fed in the system, the respective output is comprised of the same harmonics as the input. Whereas in a nonlinear system, other harmonics of the input are observed in the output of the system. ${ }^{4,5}$ It is also the same in a loudspeaker that is driven in a nonlinear working condition. Nonlinear behaviour can be 


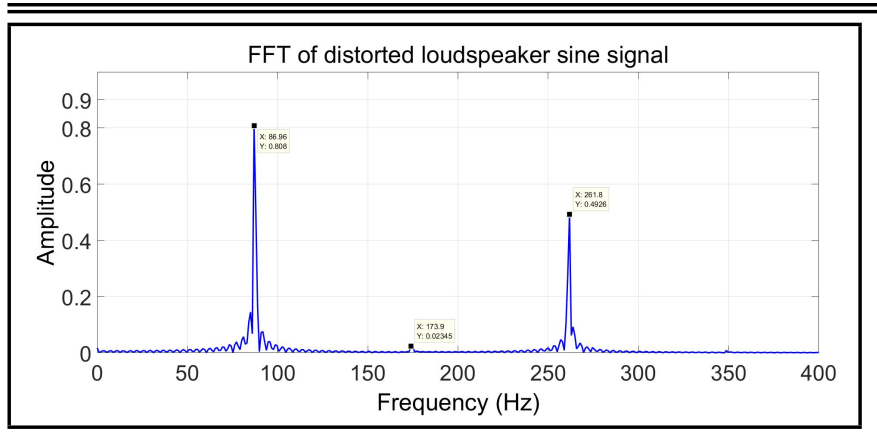

Figure 1. Distorted sine signal input of $87 \mathrm{~Hz}$, measured in output of nonlinear loudspeaker.

easily revealed in fast Fourier transform (FFT) plots. In our case, the loudspeaker shows such response as illustrated in Fig. 1.

In this figure, the first peak is associated with the input signal of $87 \mathrm{~Hz}$ frequency, and in addition, there are two sub-harmonics that are involved in the output in approximately $172 \mathrm{~Hz}$ and $262 \mathrm{~Hz}$, the peak values of which are less than the first harmonic.

The nonlinear behaviour can be mathematically expressed, as well. A mathematical model of a nonlinear loudspeaker was introduced by Ravaud et al. as Eq. (1). ${ }^{4}$

$$
a(i) \frac{d^{3} x(i)}{d t^{3}}+b(i) \frac{d^{2} x(i)}{d t^{2}}+c(i) \frac{d x(i)}{d t}+d(i)=u(t)
$$

in which $x$ is the displacement of loudspeaker diaphragm, $u$ is the input voltage of the loudspeaker, and $a, b, c$, and $d$ are the coefficients that vary with the input current and consequently, the response will be rendered nonlinear.

\section{EXPERIMENTAL SETUP}

In this paper, the identification process is performed on a Behringer loudspeaker with 8 ohms as impedance and 25 volts as maximum input voltage. This instrument is driven using an Advantech PCI 1710 data acquisition card, with an integration with intermediate circuits, such as a STK439 amplifier. In order to measure the displacement of the diaphragm of the loudspeaker, a ZS-LD80 OMRON Laser Doppler Vibrometer (LDV) was utilized. In fact, the output is the velocity of the diaphragm and an integrator can be used to estimate displacement. Schematic diagram of the circuit of identification setup is illustrated in Fig. 2.

As shown in this figure, using MATLAB, white noise signal is sent to the DAQ card with 4000 sample/sec rate and then is handed to an amplifier, the output of which is the input of the loudspeaker. This signal is sampled by the data acquisition (DAQ) card, as well. Simultaneously, laser sends position data to the DAQ card, which is saved in the computer's memory. In other words, loudspeaker input voltage as well as its diaphragm's relevant displacement voltage is saved at the same time. In this way, the input signal of loudspeaker used for identification would not be contaminated by the dynamics of the amplifier. The input voltage is also changed in order to sample different working conditions for the loudspeaker, i.e. linear and nonlinear response of the system. The saved data will be used in Sec. 5 .

\section{IDENTIFICATION OF LOUDSPEAKER}

As mentioned in Sec. 1, in some cases, it is necessary to mathematically identify loudspeakers in order to incorporate the mathematical model somewhere in processes. The identification of the nonlinear behaviour of a loudspeaker is the main goal in this section. An important issue in the identification of the loudspeaker in this paper is the robustness of the models. Considering the fact that in linear systems the frequency and amplitude of the output are proportional to the input attributes, which is not true in nonlinear systems, the robustness of identified model with regard to the input of the system develops into an important issue. As a result, the methods of identification are selected here in a way to fulfil this need.

\subsection{Methods of Identification}

First step in all system identification techniques is selecting the appropriate model structure. The characteristics such as linearity or nonlinearity and the complexity of the model should be selected in this stage.

Due to the inevitable necessity of knowing nonlinear dynamics of the loudspeaker for active noise cancellation application, dynamics of this system is identified in nonlinear regime with several methods, and the best method among them is selected based on the robustness and simplicity criteria. Also note that decision about the order of model structure is optimized with trial and error procedure and MATLAB system identification toolbox is used in the identification process. Considering the nonlinearity of the response of the system, the following methods are selected as candidates capable of following this manner.

\section{i. Prediction error method (PEM);}

ii. Nonlinear autoregressive network with exogenous inputs (NARX) with:
a) Sigmoidnet,
b) Wavenet,
c) Treepartition nonlinear functions,

iii. Series-parallel nonlinear autoregressive network with exogenous inputs (NARXSP),

Among these methods, PEM is used to identify linear systems and it is brought here to demonstrate its incapability of identifying nonlinearities, which, on the contrary, other methods should be able of identifying them. Here, the brief descriptions about these methods, which are used in this paper, are presented.

\subsection{Prediction Error Method (PEM)}

In the PEM, the base goal is to find the model parameters $\theta$, which best predicts the outcome of the system. The method can be used on linear or nonlinear model structures with an arbitrary order of difference equation. However, using this method is common on linear regressors such as (ARX and ARMAX) due to its simplicity. In the general form, the prediction error is defined as:

$$
\varepsilon(t, \theta)=y(t)-\hat{y}(t \mid t-1, \theta)
$$




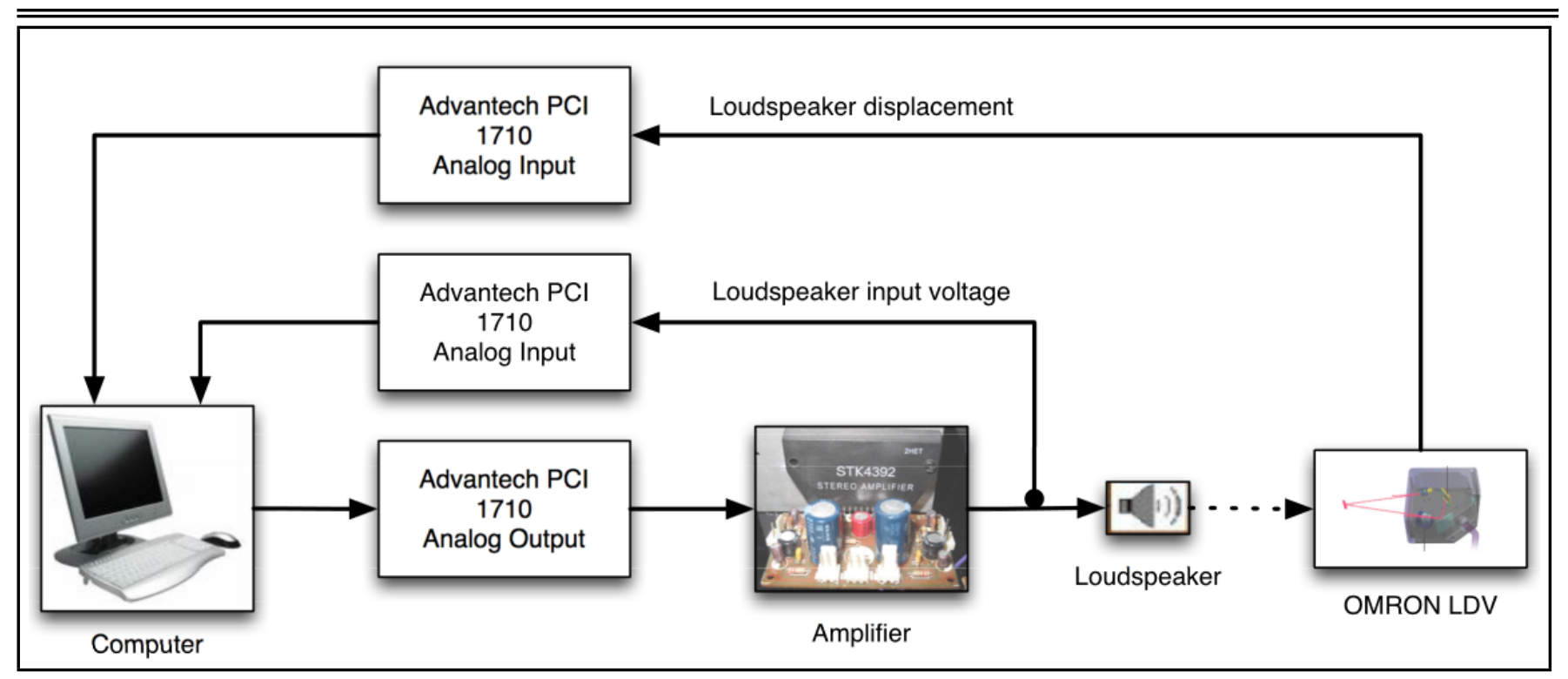

Figure 2. Experimental system setup schematic circuit.

where $y(t)$ is the real output and $\tilde{y}(t \mid t-1, \theta)$ is the predicted output. The prediction error method encompasses of finding the parameters $\theta$ that makes the prediction errors as small as possible. As mentioned $i^{20}$ a general linear prediction is defined by:

$$
\hat{y}(t \mid t-1, \theta)=L_{1}(q, \theta) y(t)+L_{2}\left(q^{-1}, \theta\right) u(t) ;
$$

in Eq. (3) $L_{1}$ and $L_{2}$ are difference operators composing estimator model. The order of this model should be decided based on its performance and using trial and error procedure, but the parameters or coefficients of the model are designed to minimize the prediction error. The cost function used for optimal estimation of parameters $\theta$ is the mean square of the prediction error, which is defined as:

$$
V_{N}(\theta)=\frac{1}{N} \sum_{t=1}^{N} \varepsilon^{2}(t, \theta), \hat{\theta}=\arg \min _{\theta} V_{N}(\theta) ;
$$

details about this method can be found in. ${ }^{20}$ But it is worth mentioning that optimization problem in Eq. (4) has a closed form solution for linear structure models such as (ARX and ARMAX), and commercial software for example MATLAB can solve it quickly.

\subsection{Nonlinear ARX (NARX)}

There are many sources of nonlinear behaviour in the loudspeaker dynamics. Hence, the importance of nonlinear model identification methods is obvious. Classical methods in this category are based on polynomial approximations of the model structure. Some of the special structures for nonlinear systems are Hammerstein and Wiener models, ${ }^{21,22}$ which separate primary nonlinear structure to (nonlinear-linear) and (linearnonlinear) series sub structures respectively.

The other common structure in the nonlinear system identification is NARX structure. This structure is an extension of the linear ARX structure. The structure is formed by a linear and a nonlinear function in parallel arrangement, which is explained in the Fig. 3.

In the mathematical form the output can be explained as:

$$
y=L^{T}(X-r)+g(Q(X-r)) ;
$$

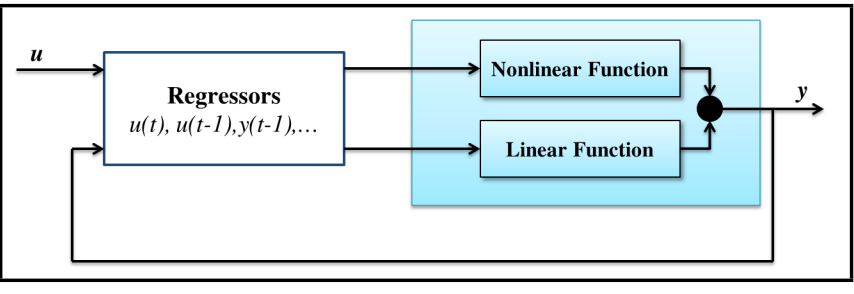

Figure 3. Nonlinear ARX (NARX) structure.

where in Eq. (5) $X, r, Q$ are vector of regressors, mean of the regressors and projection Tmatrix, respectively. Also $L^{T}$ and $g$ are linear and nonlinear functions in Fig. 3, respectively. ${ }^{23}$ Most of the nonlinear functions used as $g$ in NARX structure are composed of limited number of nonlinear units such as wavelet network, sigmoid network, or tree- partition network. Fortunately, all of these nonlinear functions exist in MATLAB system identification toolbox. In this paper, loudspeaker nonlinear dynamics is identified with these nonlinear functions and a comparison between them is done. Details about these functions are explained in. ${ }^{23}$

\subsection{Neural Network}

As proved in a previous study, ${ }^{24}$ neural networks are universal approximators for static nonlinearities and are consequently a good alternative for polynomial approaches Furthermore, memoryless static networks can be extended with dynamic components to dynamic neural networks; hence, they can be used in nonlinear system identification. ${ }^{25,26}$ One of the structures of neural network that can be used in the offline identification of nonlinear system is series-parallel structure, which will be used in this paper. Due to the popularity of neural network application in approximating the systems behaviour, in this paper, explanations are restricted only to series parallel network. More details about the neural networks and series-parallel structure can be found in previous studies. $^{24,27,28}$ Series-parallel network uses actual system input and output with specified order of unit delay operator as shown in the Fig. 4.

Also the mathematical representation of the output after 


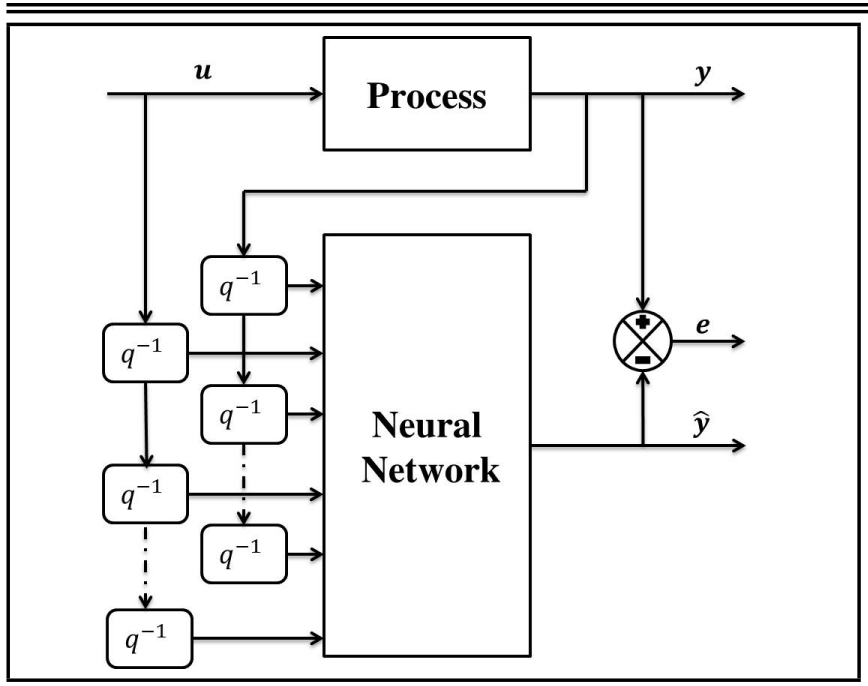

Figure 4. Series-parallel NARX.

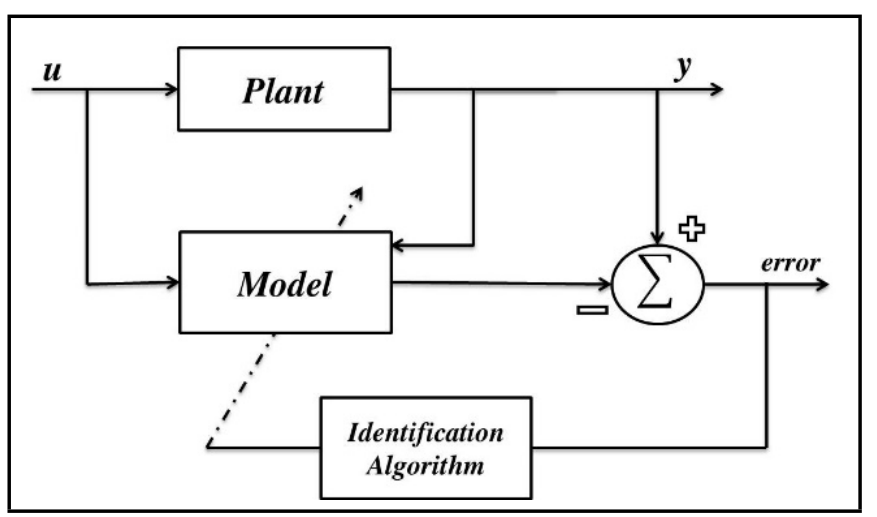

Figure 5. Generic structure of system identification.

training process is given by:

$$
\hat{y}(k)=f_{N N}(u(k), u(k-1), \ldots, y(k-1), y(k-2), \ldots) ;
$$

as mentioned before, due to the offline identification procedure, which is used in this paper, the series parallel structure is very useful and can be used easily. Furthermore, the number of neurons and layers are selected with trial and error process, and Levenberg Marquardt method is used to optimize the error of estimation in training phase.

\subsection{Input/output Criteria}

In this article, all of the aforementioned methods are applied on the loudspeaker data. Identification procedure is done with white noise input signal due to its excitation persistency, and test phase is done with pure sine wave inputs with different amplitudes and frequencies to study the robustness of the methods.

In order to do so, the following structure in Fig. 5 is utilized, and it should be emphasized that identification process is an offline one in this paper i.e. identification is carried out as a post process on the data already saved in the computer memory. In the Fig. 5, u is white noise input with maximum amplitude equals to 15 volts and $y$ is the actual output of the loudspeaker. Input and output of the system are acquired with a sampling rate of $4000 \mathrm{sample} / \mathrm{sec}$ and for $1 \mathrm{sec}$. In other words, only 4000 data are saved for offline identification.

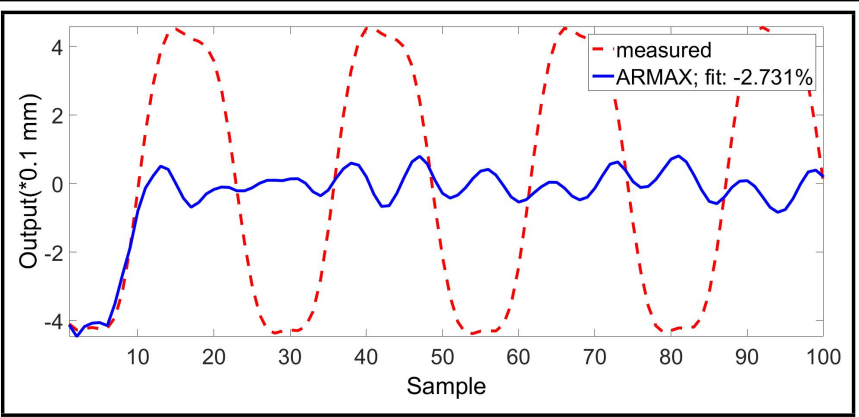

Figure 6. Performance of ARX model under sine input with high magnitude (20 volts) and frequency of $155 \mathrm{~Hz}$.

Table 1. Characteristics of the piezoelectric layers and flexible appendages. ${ }^{14}$

\begin{tabular}{|c|c|c|}
\hline$n_{a}$ & $n_{b}$ & $n_{c}$ \\
\hline 55 & 55 & 55 \\
\hline
\end{tabular}

\section{RESULTS}

The results of identification are divided into three groups, one for each method described in Sec. 4.

\subsection{Identification Using PEM}

A PEM method with the properties as indicated in Table 1 is used to best fit the input/output of the system.

Parameters in Table 1 are degrees of numerator, denominator of input/output filter model, and the denominator of noise filter model, respectively. Having performed the identification process, the fitness of PEM method on the nonlinear response of loudspeaker was turned out to be $78.94 \%$ for white noise, which is good for a linear identifier in a nonlinear system. However, this is to remind that the resulting transfer function would have a degree of 55, as shown in Table 1, which exerts a huge amount of computational load on the processor. Moreover, the test result illustrated in Fig. (6) shows that PEM is not suitable choice for this nonlinear system.

\subsection{Identification Using NARX}

For the NARX method, the system that best follows the nonlinear behaviour of the loudspeaker is described in Table 2, where parameters in Table 2 are NARX model parameters in MATLAB Identification Toolbox. Having performed the identification process, the fitness of NARX method on the nonlinear response of loudspeaker with white noise input turned out to be $83.54 \%, 71.54 \%$, and $90.8 \%$ for sigmoid, treepartition, and wavenet functions, respectively . Comparing Table 2 with Table 1 and taking into consideration the fitness values of abovementioned methods and nonlinear functions, it is observed that NARX method exhibits approximately the same results with a very lower model degree. In order to study the robustness of the proposed methods regarding input amplitude and frequency uncertainties, designed NARX models are tested with two pure sine inputs. Figure 7 and 8 show the performance of the aforementioned NARX models.

Table 2. Characteristics of the piezoelectric layers and flexible appendages. ${ }^{14}$

\begin{tabular}{||c|c|c|c|c||}
\hline Nonlinear Function & $n_{a}$ & $n_{b}$ & $n_{k}$ & Number of units \\
\hline Sigmoid & 5 & 5 & 5 & 10 \\
\hline Treepartition & 8 & 8 & 8 & 255 \\
\hline Wavenet & 12 & 12 & 12 & 17 \\
\hline
\end{tabular}




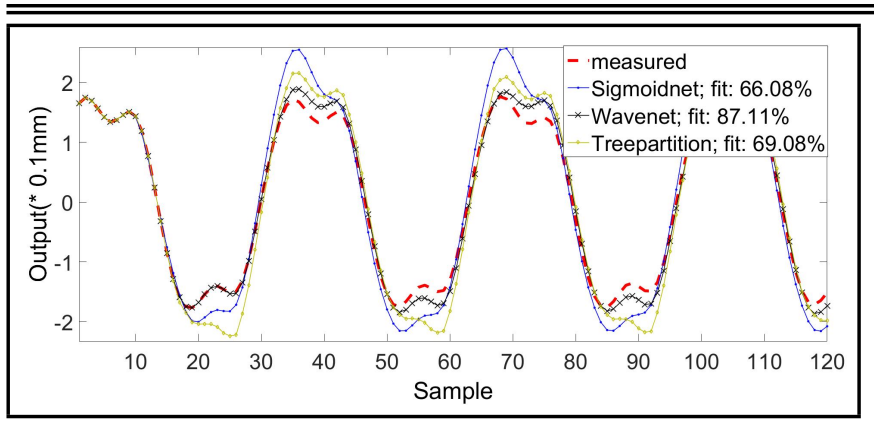

Figure 7. Performance of NARX model under sine input with low magnitude (10 volts) and frequency of $121 \mathrm{~Hz}$.

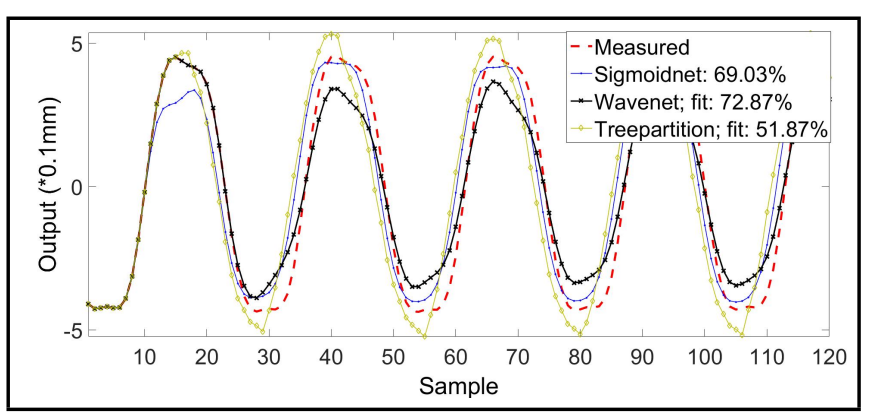

Figure 8. Performance of NARX model under sine input with high magnitude (20 volts) and frequency of $155 \mathrm{~Hz}$.

Based on Fig. 7 and Fig. 8, wavenet best followed nonlinear response of the loudspeaker in both of the tests. Especially for high magnitude and frequency input, Fig. 8 shows that treepartition model had a huge amount of error.

\subsection{Identification Using Series-Parallel NARX}

For the NARXSP method, the system that best follows the nonlinear behaviour of the loudspeaker is a neural network with three layers, which has 10,10 , and 5 neurons in the first, second, and third layer, respectively. The training method is also chosen to be Levenberg Marquardt. In addition, the number of delays is four samples. The nonlinear function of every neuron in hidden layers is hyperbolic tangent sigmoid, too.

Having performed the identification process, the performance of NARXSP method on nonlinear response of loudspeaker was turned. The training error for white noise input is illustrated in Fig. 9, which shows its extreme smallness. Also, the robustness of this model is tested with the previous sine inputs in Fig. 10 and Fig. 11. Figure 10 and 11 demonstrate the series parallel model ability in facing input uncertainties. Comparison of Fig. 7 with Fig. 10 and Fig. 8 with Fig. 11 depicts the advantages and the better performance of seriesparallel NARX model in comparison with conventional NARX model. This advantage can be related to neural network ability in modelling nonlinear functions. Therefore, this structure is the most suitable choice for noise attenuation application.

\section{CONCLUSIONS}

One of the important and contemporary applications of loudspeakers is in noise attenuation and noise cancellation. However, in order to get high performance, it is a vital need to model loudspeaker nonlinear dynamics. Therefore, in this article different methods to identify the nonlinear dynamic of

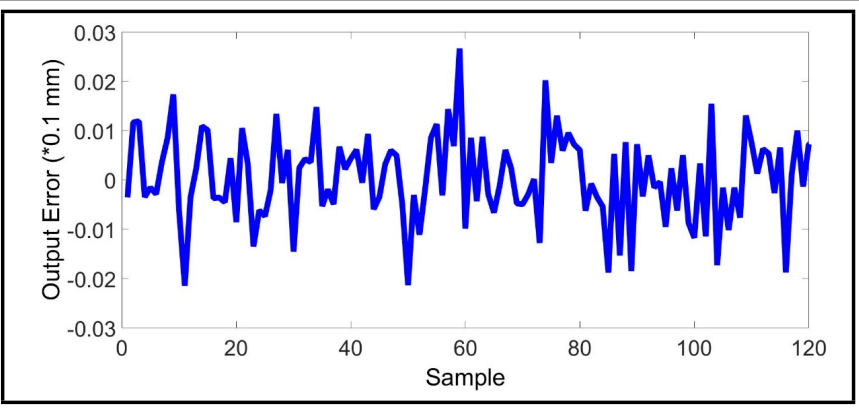

Figure 9. Training error of series-parallel NARX.

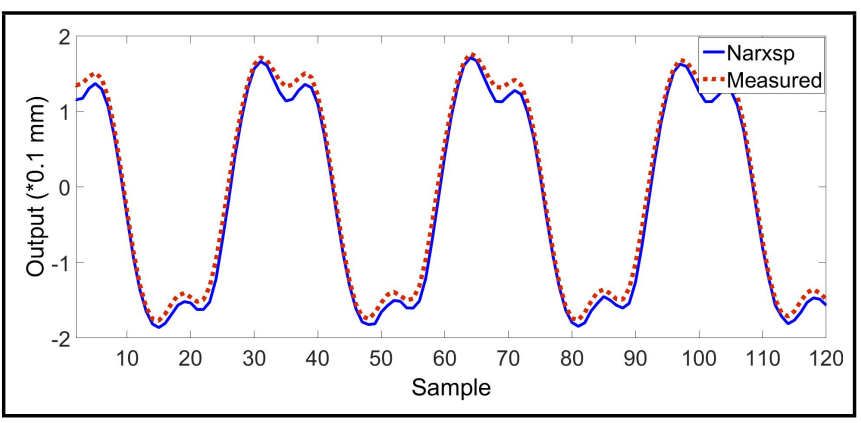

Figure 10. Performance of series-parallel NARX model under low magnitude (10 volts) sine input with frequency of $121 \mathrm{~Hz}$.

loudspeaker were studied. In fact, PEM, NARX, and seriesparallel NARX methods were utilized to model the relation between input voltage and the displacement of the loudspeaker diaphragm in nonlinear regime. White noise was used to train these models and pure sine inputs with a variety of amplitudes and frequencies were exerted to examine model performance regarding input uncertainty. The results of this study demonstrated that series-parallel NARX model, which utilized neural network, was the best choice to model loudspeaker dynamics. Furthermore, NARX model with wavenet block was the second appropriate choice after neural network. Finally, linear model even with a high degree could not be robust against input variations.

\section{REFERENCES}

1 Thiele, A. N. Loudspeakers in vented boxes: Part 1and 2, Vol 1, Audio Eng. Soc., New York, (1978).

2 Borwick, J. Loudspeaker measurements, Loudspeaker and Headphone Handbook, Focal Press, Oxford, (2001).

3 Klippel, W. Loudspeaker nonlinearities - cause, parameters, symptoms, J. Audio Eng. Soc., 54, 907-939, (2006).

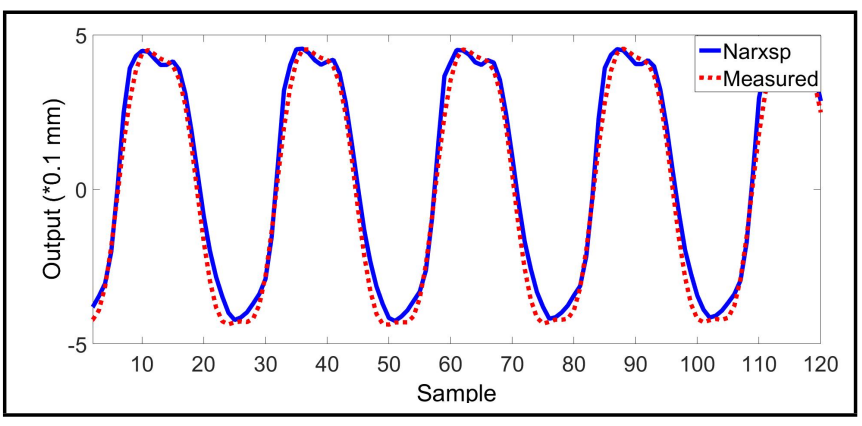

Figure 11. Performance of series-parallel NARX model under high magnitude (20 volts) sine input with frequency of $155 \mathrm{~Hz}$. 
${ }^{4}$ Ravaud, R., Lamarquand, G. and Roussel, T. Timevarying nonlinear modeling of electrodynamic loudspeakers, Applied Acoustics, 70, 450-458, (2009). https://dx.doi.org/10.1016/j.apacoust.2008.05.009

5 Ravaud, R. Lemarquand, G. Roussel, T. and Lemarquand, V. Ranking of the nonlinearities of electrodynamic loudspeakers, Acoustics, 35, 49-66, (2010). https://dx.doi.org/10.2478/v10168-010-0004-6

${ }^{6}$ Faifer, M. Ottoboni, R. and Toscani, S. A novel, costeffective method for loudspeakers parameters measurement under non-Linear conditions, IEEE AUTOTESTCON COnference, Salt Lake City, UT, (2008).

${ }^{7}$ Chang, C. Pawar, S.J. Soar, W. Shiah, Y.C and Huang, J.H. Effect of nonlinear stiffness on the total harmonic distortion and sound pressure level of a circular miniature loudspeaker-experiments and simulations, IEEE Transaction of Consumer Electronics, 58 (2), 212-220, (2012). https://dx.doi.org/10.1109/TCE.2012.6227415

8 Chang, C. Wang, C.C. Shiah, Y.C. and Huang, J.H. Numerical and experimental analysis of harmonic distortion in a moving-coil loudspeaker, Communications in Nonlinear Science and Numerical Simulation, 18 (7), 1902-1915, (2013). https://dx.doi.org/10.1016/j.cnsns.2012.11.021

9 Veprik, A. M. Vibration protection of critical components of electronic equipment in harsh environmental conditions. Journal of Sound and Vibration, 259 (1), 161-175, (2003). https://dx.doi.org/10.1006/jsvi.2002.5164

${ }^{10}$ Ho, V. C., Veprik, A. M., and Babitsky, V. I. Ruggedizing printed circuit boards using a wideband dynamic absorber. Shock and Vibration, 10 (3), 195-210, (2003). https://dx.doi.org/10.1155/2003/185798

${ }^{11}$ Hosseinloo, A. H., Yap, F. F., and Lim, L. Y. Design and analysis of shock and random vibration isolation system for a discrete model of submerged jet impingement cooling system. Journal of Vibration and Control, 21 (3), 468-482, (2015). https://dx.doi.org/10.1177/1077546313490186

12 Hosseinloo, A. H., Tan, S. P., Yap, F. F., and Toh, K. C. Shock and vibration protection of submerged jet impingement cooling systems: Theory and experiment. Applied Thermal Engineering, 73 (1), 1076-1086, (2014). https://dx.doi.org/10.1016/j.applthermaleng.2014.08.059

${ }^{13}$ Hosseinloo, A. H., Yap, F. F., and Chua, E. T. Random vibration protection of a double-chamber submerged jet impingement cooling system: A continuous model. Aerospace Science and Technology, 35, 29-38, (2014). https://dx.doi.org/10.1016/j.ast.2014.02.005

${ }^{14}$ Hosseinloo, A. H., Yap, F. F., and Vahdati, N. Analytical random vibration analysis of boundary-excited thin rectangular plates. International Journal of Structural Stability and Dynamics, 13 (03), 1250062, (2013). https://dx.doi.org/10.1142/S0219455412500629

15 Veprik, A. M., Babitsky, V. I., Pundak, N., and Riabzev, S. V. Vibration protection of sensitive components of infrared equipment in harsh environments. Shock and Vibration, 8 (1), 55-69, (2001). https://dx.doi.org/10.1155/2001/501572

16 Oh, H. U., Lee, K. J., and Jo, M. S. A passive launch and on-orbit vibration isolation system for the space borne cryocooler. Aerospace Science and Technology, 28 (1), 324331, (2013). https://dx.doi.org/10.1016/j.ast.2012.11.013

17 Azadi, N. and Ohadi, A.R. Enhanced Multi-Channel Active Fuzzy Neural Network Noise Control in an Enclosure, Proceedings of the World Congress on Engineering (WCE), Vol III, London, UK, (2011).

18 Azadi, N. and Ohadi, A.R. Filtered gradient active fuzzy neural network noise control in an enclosure backed by a clamped plate, Int. Journal of Adaptive Control and Signal Processing, 26 (5), 451-468, (2012). https://dx.doi.org/10.1002/acs.1298

${ }^{19}$ Sjberg, J. Zhang, Q. Ljung, L. Benveniste, A. Delyon, B. Glorennec, P.Y. Hjalmarsson, H. and Juditsky, A. Nonlinear black-box modeling in system identification: a unified overview, Automatica, 31 (12), 1691-1724, (1995). https://dx.doi.org/10.1016/0005-1098(95)00120-8

20 Ljung, L. System identification: Theory for the user, 2nd Ed., Prentice Hall, Upper Saddle River, NJ, (1999). https://dx.doi.org/10.1002/047134608x.w1046

${ }^{21}$ Haber, R. and Unbehauen, H. Structure identification of nonlinear dynamic systems: A survey on input/output approaches, Automatica, 26 (4), 651-677, (1990). https://dx.doi.org/10.1016/0005-1098(90)90044-I

${ }^{22}$ Liu, Y. and Bai, E.W. Iterative identification of Hammerstein systems, Automatica 43 (2), 346-354, (2007). https://dx.doi.org/10.1016/j.automatica.2006.09.004

${ }^{23}$ Ljung, L. The System Identification Toolbox: The manual, 6th Ed., MathWorks Inc., Natick, MA, (2003).

${ }^{24}$ Narendra, S.K. and Parthasarathy, K. Identification and control of dynamical systems using neural networks, IEEE Transaction on Neural Network, 1 (1), 4-27, (1990). https://dx.doi.org/10.1109/72.80202

25 Nelles, O. Nonlinear system identification: From classical approaches to neural networks and fuzzy models, Springer, Berlin, (2001).

26 Isermann, R. Ernst, T.S and Nelles, O. Identification with dynamic neural networks: architecture, comparisons, applications, Proceedings of the 11th IFAC Symposium on System Identification, Fukuoka, Japan, (1997).

27 Isermann, R. and Munchhof, M. Identification of dynamic systems: An introduction with application, Springer, Berlin, (2011).

28 Demuth, H. Beale, M. Hagan, M. Neural Networks Toolbox User's Guide, 4th Version, MathWorks Inc., Natick, MA, (2005). 